\title{
v-Ha-ras Mitogenic Signaling Through Superoxide and Derived Reactive Oxygen Species
}

\author{
Ji-Qin Yang, ${ }^{1}$ Garry R. Buettner, ${ }^{1}$ Frederick E. Domann, ${ }^{1}$ Qiang Li, ${ }^{2}$ John F. Engelhardt, ${ }^{2}$ \\ Christine Darby Weydert, ${ }^{1}$ and Larry W. Oberley ${ }^{1 *}$ \\ ${ }^{1}$ Free Radical and Radiation Biology Program, Department of Radiation Oncology, B180 Medical Laboratories, University of \\ Iowa, Iowa City, Iowa \\ ${ }^{2}$ Department of Anatomy and Cell Biology, University of Iowa, Iowa City, Iowa
}

The ras proto-oncogene is frequently mutated in human tumors and functions to constitutively stimulate signal transduction cascades, resulting in unchecked proliferation and malignant transformation. In certain cells, superoxide functions as a signal-transduction messenger, mediating the downstream effects of ras and rac. We demonstrated previously that v-Ha-ras-transfected rat kidney epithelial cells (RECs) overproduced superoxide anion and that this superoxide production was mediated by ras. In the present study, we further demonstrated that v-Ha-ras overexpression transformed immortal nonmalignant RECs into malignant cancer cells; v-Ha-ras-transfected cells formed clones in soft agar, had high plating efficiency, and formed tumors in nude mice. Our data suggest that superoxide radical plays a role in ras-induced transformation; modulation of intracellular superoxide level by overexpression of manganese-containing superoxide dismutase or copper- and zinc-containing superoxide dismutase inhibited ras-induced transformation, as evidenced by in vitro studies of plating efficiency and by in vivo studies of tumor formation in nude mice. Overexpression of catalase (CAT) alone was found to have little effect on tumor cell growth, but overexpression of glutathione peroxidase 1 (GPx1) completely suppressed tumor cell growth in nude mice. This finding suggests that peroxides removed by GPX1, but not by CAT, are also involved in ras-induced transformation. () 2002 Wiley-Liss, Inc.

Key words: free radicals; redox; superoxide dismutase; catalase; glutathione peroxidase; signal transduction

\section{INTRODUCTION}

The ras oncogene plays an important role in mitogenesis induced by growth factors. Approximately $30 \%$ of all human tumors contain mutated versions of ras proteins. In some tumors (e.g., carcinomas of the colon, pancreas, and thyroid), the incidence of ras mutation is even higher [1]. Mutation of the ras gene is the single most common abnormality of dominant oncogenes in human tumors and is extremely common in carcinogenesis.

Reactive oxygen species (ROS), including superoxide radical, hydroxyl radical, and hydrogen peroxide, have a higher reactivity than that of ground-state molecular oxygen and are generated during the course of normal aerobic respiration [2]. To protect cells against ROS, cells are equipped with antioxidant enzymes. Superoxide dismutase (SOD) is one of the primary antioxidant enzymes. There are three isoforms of SOD in human cells: copperand zinc-containing superoxide dismutase (CuZnSOD), which is found predominantly in the cytoplasm; manganese-containing superoxide dismutase (MnSOD), which exists primarily in the mitochondrial matrix; and extracellular SOD, which is secreted. This family of enzymes catalyzes the dismutation of superoxide to dioxygen and hydrogen peroxide. Hydrogen peroxide is further detox- ified to water by the enzymes catalase (CAT) and glutathione peroxidase (GPx). Therefore, through these proteins, ROS levels are strictly regulated by cells.

A number of studies have demonstrated that, in general, cancer cells have lowered MnSOD and CuZnSOD activity as compared with the normal cell type from which the tumor arose [3-5]. This observation has been made in a number of cell types and animal species and is independent of the mechanism of cell transformation [6-9]. Cancer cells not only exhibit low constitutive MnSOD

*Correspondence to: Free Radical and Radiation Biology Program, B180 Medical Laboratories, Department of Radiation Oncology, University of lowa, lowa City, IA.

Received 11 September 2001; Revised 10 October 2001; Accepted 16 January 2002

Abbreviations: AdCAT, adenovirus-catalase construct; AdCuZn$S O D$, adenovirus-copper and zinc-containing superoxide dismutase construct; AdGPx1, adenovirus-glutathione peroxidase1 construct; $\operatorname{Ad} L a c Z$, adenovirus- $\beta$-galactosidase construct; $A d M n S O D$, adenovirus-manganese-containing superoxide dismutase construct; $A P-1$ activator protein 1; CAT, catalase; CUZnSOD, copper- and zinccontaining superoxide dismutase; GPx, glutathione peroxidase MnSOD, manganese-containing superoxide dismutase; MOI, multiplicity of infectivity; NAC, N-acetylcysteine; NF- $\mathrm{B}$, nuclear factor $\kappa \mathrm{B}$; REC, rat kidney epithelial cell; ROS, reactive oxygen species; SOD, superoxide dismutase.

DOI 10.1002/mc.10037 
levels but, at least in some cancer cell types, have also lost the ability to undergo induction of this protein by oxygen or superoxide exposure [10]. Many recent studies have focused on the tumorsuppressive effects of MnSOD [11-15]. Similar results among these investigations have indicated that increased levels of MnSOD protein suppress the malignant phenotype, as evidenced by slower cell growth rate, lower colony formation both in normal medium and in soft agar, and less tumor formation in nude mice, as compared with the parental untransfected cells.

ROS have been regarded as having carcinogenic potential and have been associated with tumor promotion [16]. In recent years, it has been hypothesized that cells can regulate signal transduction pathways and alter the expression of certain genes by altering ROS levels. It has also been shown that ROS act as intracellular secondary messengers for several cytokines and growth factors [17-21]. Thus, ROS at low concentrations are now thought of as signaling molecules, but at high concentrations, ROS are toxic. v-Ha-ras overexpression can induce superoxide generation in human fibroblasts [22] and in human keratinocyte HaCaT cells [23]. We previously demonstrated that superoxide is overproduced in v-Ha-ras-transfected rat kidney epithelial cells (RECs) [24]. In the present study, we found that $\mathrm{v}$-Ha-ras overexpression could malignantly transform REC cells. Moreover, by overexpressing MnSOD, CuZnSOD, or GPx1, but not CAT, scavenging superoxide or superoxide-derived ROS could inhibit mitogenic signal transduction and malignant transformation mediated by v-Ha-ras. Thus, these data suggest that superoxide and ROS derived from superoxide play an essential role in v-Ha-ras mitogenic signaling and malignant transformation.

\section{MATERIALS AND METHODS}

\section{Cell Lines and Cell Culture}

The immortal nonmalignant rat kidney epithelial cell line NRK52E was obtained from the American Type Culture Collection (Rockville, MD; cell line CRL-1571) [25]. Cells were maintained in highglucose Dulbecco's modified Eagle's medium containing 5\% bovine calf serum, $2 \mathrm{mM}$ L-glutamine, and $0.1 \mathrm{mM}$ nonessential amino acids at $37^{\circ} \mathrm{C}$ in $5 \%$ $\mathrm{CO}_{2}$. Cells were fed three times a week. v-Ha-rastransfected RECs were also cultured in $400 \mu \mathrm{g} / \mathrm{mL}$ G418, which was removed $3 \mathrm{~d}$ before an experiment. These cell lines have been described elsewhere [24]. The cells used in our experiments were from passages 10-40 after procurement from the American Type Culture Collection.

\section{SOD, CAT, and GPx Activity Gel Assay}

Cells were harvested and lysed by sonication; protein concentrations were then quantitated with the Bradford protein assay kit (Bio-Rad, Hercules, CA). SOD, CAT, and GPx activities were measured with a native gel activity stain [26,27]. An $8 \%$ running gel and 5\% stacking gel were used to separate the proteins. For the SOD activity stain, the gel was stained with $2.43 \mathrm{mM}$ nitroblue tetrazolium solution for $20 \mathrm{~min}$ and then $28 \mu \mathrm{M}$ riboflavin with $28 \mathrm{mM} \mathrm{N}, \mathrm{N}, \mathrm{N}^{\prime}, \mathrm{N}^{\prime}$-tetramethylethylenediamine for $15 \mathrm{~min}$. The gel was placed in distilled water and illuminated under a bright fluorescent light. Achromatic bands indicated the presence of SOD. For the CAT activity stain, the gel was first rinsed three times with distilled water, followed by incubation in $0.003 \% \mathrm{H}_{2} \mathrm{O}_{2}$ for $10 \mathrm{~min}$. The gel was then stained with $2 \%$ ferric chloride and $2 \%$ potassium ferricyanide; when achromatic bands began to form, the stain was poured off and the gel rinsed extensively with distilled water. Achromatic bands demonstrated the presence of catalase activity. A similar procedure was used for GPx activity [27]. Gels were first soaked in three changes of reduced glutathione for a total of $45 \mathrm{~min}$ and then soaked in $0.008 \%$ cumene hydroperoxide plus glutathione for $10 \mathrm{~min}$. The gels were rinsed twice with distilled water and then stained with ferric chloride and potassium ferricyanide, as described above. In this study, $100 \mu \mathrm{g}$ of total protein from cell lysates was run in each lane for the SOD gels, $200 \mu \mathrm{g}$ for the CAT gels, and $400 \mu \mathrm{g}$ for the GPx gels.

\section{Plating Efficiency}

Cells were plated in triplicate into $60-\mathrm{mm}$ dishes in complete media. The number of cells plated was 100 or 500 . The dishes were maintained in the incubator for $14 \mathrm{~d}$ to permit colony formation. The colonies were then fixed and stained with $0.1 \%$ crystal violet and $2.1 \%$ citric acid, and those colonies containing $>50$ cells were scored.

Infection with Adenovirus-Expressing MnSOD, CUZnSOD, CAT, or GPX1

The adenovirus constructs used were replicationdefective, E1-deleted recombinant adenoviruses. Human MnSOD cDNA, human CuZnSOD cDNA, human CAT cDNA, human GPX1 cDNA, or lacZ cDNA was inserted into the E1 region of the viral genome; these foreign genes were driven by a cytomegalovirus (CMV) promoter [28,29]. GPx1expressing recombinant adenovirus was made as recently described [29]. The construct contains the human GPX1 cDNA with a c-myc tag at the $\mathrm{N}$ terminus of the protein [29]. Approximately $2 \times 10^{5}$ cells from a v-Ha-ras-overexpressing REC cell line (U23) [24] were seeded in six-well plates and left to adhere for $12 \mathrm{~h}$. Cells were then washed three times in serum-free media. The adenovirus-MnSOD (AdMnSOD), adenovirus-CuZnSOD (AdCuZnSOD), adenovirus-CAT (AdCAT), or adenovirus-GPx1 
(AdGPx1) constructs were added to cells in $2 \mathrm{~mL}$ of serum-free medium at the indicated multiplicity of infection (MOI). The U23 cell line was treated with the adenovirus-lacZ ( $\mathrm{d} \mathrm{L} L a c Z$ ) construct as a control. Cells were incubated with adenovirus constructs for $12 \mathrm{~h}$. Serum-free medium was replaced with complete medium for an additional $24 \mathrm{~h}$ before cells were assayed for activity and immunoreactive protein.

\section{Native GPx1 Immunoblotting Assay}

We developed an immunoblotting method to determine the GPx1 protein levels in different clones [30]. The protein separation method was the same as for the GPx activity gel assay described above. The native proteins were transferred onto the nitrocellulose membrane by the same method as in a Western blotting assay. The detection method was also the same as that for the Western blotting. A GPx1 primary antibody produced in our laboratory was diluted 1:100.

\section{Gel Mobility Shift Assay}

Nuclear protein was extracted by the following procedure [31]. Cells were collected when they reached $80 \%$ confluence and were rinsed with phosphate-buffered saline (PBS). Cells were then harvested by scraping in the presence of ice-cold buffer A (10 mM HEPES, $1.5 \mathrm{mM} \mathrm{MgCl}_{2}$, and $10 \mathrm{mM}$ $\mathrm{KCl}$ ) and incubated on ice for $20 \mathrm{~min}$. The cells were lysed with a dounce homogenizer (Kontes, Vineland, $\mathrm{NJ}$ ); the nuclei were pelleted by centrifuging twice at $5,000 \mathrm{~g}$ for $30 \mathrm{~s}$ at $4{ }^{\circ} \mathrm{C}$, with subsequent removal of supernatant. The nuclear pellets were resuspended in ice-cold buffer $\mathrm{C}$ (20 mM HEPES, $25 \%$ glycerol, $0.42 \mathrm{M} \mathrm{NaCl}, 1.5 \mathrm{mM} \mathrm{MgCl}_{2}, 0.2 \mathrm{mM}$ EDTA, and $0.5 \mathrm{mM}$ phenylmethylsulfonyl fluoride) and placed on ice for $15 \mathrm{~min}$. The suspensions were centrifuged and the supernatants removed and diluted with ice-cold buffer D (20 mM HEPES, 20\% glycerol, $0.1 \mathrm{M} \mathrm{KCl}, 0.2 \mathrm{mM}$ EDTA, and $0.5 \mathrm{mM}$ phenylmethylsulfonyl fluoride). The protein concentrations were determined using the Bio-Rad Bradford dye binding protein assay kit according to the manufacturer's instructions. The nuclear protein extracts were stored at $-80^{\circ} \mathrm{C}$.

DNA binding reactions were carried out with $1.5 \mu \mathrm{g}$ of extracted nuclear protein and doublestranded synthetic oligodeoxynucleotide encompassing the AP-1 consensus sequence $5^{\prime}$-agcttgTGACTCAccgtag-3', AP-2 consensus sequence 5'agctcaaGCCCG-CGGGCtctctag- $3^{\prime}$, or NF- $\kappa$ B consensus sequence $5^{\prime}$-agctgaGGGGACTTTCC-ctag- $3^{\prime}$. Probes were ${ }^{32} \mathrm{P}$-labeled with Klenow DNA polymerase and $\left[\alpha^{32} \mathrm{P}\right] \mathrm{dCTP}$. One and a half micrograms of nuclear protein extracts was incubated with ${ }^{32} \mathrm{P}$ labeled DNA probes in the presence of $1 \mu \mathrm{g}$ poly dIdC (Pharmacia) and $1 \times$ gel shift buffer $(10 \mathrm{mM}$
Tris, pH 7.5, $50 \mathrm{mM} \mathrm{NaCl}, 1 \mathrm{mM} \mathrm{MgCl}_{2}, 0.5 \mathrm{mM}$ EDTA, $0.5 \mathrm{mM}$ dithiothreitol, and $4 \%$ glycerol) at room temperature for $20 \mathrm{~min}$. The bound DNAprotein complexes were separated from free probe by gel electrophoresis in 5\% native polyacrylamide gels in $1 \times$ TBE ( 90 mM Tris-borate and 2 mM EDTA). Electrophoresis was conducted at a constant current of $20 \mathrm{~mA}$ for $1 \mathrm{~h}$. The gel was wrapped in a plastic wrap and exposed to X-ray film overnight at $-80^{\circ} \mathrm{C}$. AP-1 DNA-binding specificity was shown previously by competition experiments [24]. For supershift assays, $1.5 \mu \mathrm{g}$ of nuclear protein extracts were preincubated with $2 \mu \mathrm{g}$ of anti-c-jun/AP-1 or antip50 (NF-кB) antibodies (Santa Cruz Biotechnology, Santa Cruz, CA) for $30 \mathrm{~min}$ at room temperature and then incubated with ${ }^{32} \mathrm{P}$-labeled probes.

The N-acetylcysteine (NAC) experiments were done in the following manner. NAC was prepared with medium at a high concentration and the $\mathrm{pH}$ adjusted to 7.0. The solution was then filtered for sterility. The NAC solution was prepared fresh before usage and adjusted to $10 \mathrm{mM}$ final concentration in the complete tissue culture medium. After growth in this medium for $24 \mathrm{~h}$, cells were collected and nuclear protein extracted as described above.

\section{Growth in Soft Agar}

Cells were grown in T25 flasks for $48 \mathrm{~h}$, detached with $0.25 \%$ trypsin and $0.1 \%$ EDTA, and resuspended in prewarmed complete medium. Cell number was determined with a hemocytometer; aliquots of the cell suspension were mixed with 3 $\mathrm{mL}$ of medium containing $0.3 \%$ agar to yield a total cell number of $2 \times 10^{3}$ cells per dish. This was overlaid onto $3 \mathrm{~mL}$ of presolidified $0.5 \%$ agar in $60-\mathrm{mm}$ dishes. After cells were plated, the culture dishes were kept at $4^{\circ} \mathrm{C}$ for 20 min to solidify the agar and were then incubated in a $37^{\circ} \mathrm{C}$ incubator with $5 \%$ $\mathrm{CO}_{2}$ and $95 \%$ air for $14-28 \mathrm{~d}$. Colonies $\geq 1 \mathrm{~mm}$ in diameter were counted. The clonogenic fraction was calculated by

$$
\begin{aligned}
\text { Clonogenic fraction } & =\frac{\text { colonies counted }}{\text { numbers of cells seeded }} \\
& \times 100 \%
\end{aligned}
$$

\section{Growth in Nude Mice}

Five-week-old female athymic (nu/nu) mice (Harlan-Sprague-Dawley, Madison, WI) were used for the in vivo malignancy assay. The animals were kept under pathogen-free conditions at the Animal Care Facility, University of Iowa. In most cases, $5 \times 10^{5}$ cells $(0.1 \mathrm{~mL})$ were suspended in serum-free medium and inoculated subcutaneously into the rear leg. Four animals were used per group. When a tumor was palpable, it was measured by a vernier caliper once a day until the animals showed obvious signs 
of discomfort or illness. Tumor volume (TV) was calculated by the formula:

$$
\mathrm{TV}\left(\mathrm{mm}^{3}\right)=\left(\mathrm{L} \times \mathrm{W}^{2}\right) / 2
$$

where $\mathrm{L}$ is the longest dimension of the tumor (in $\mathrm{mm}$ ), and $\mathrm{W}$ is the shortest dimension of the tumor (in $\mathrm{mm}$ ) [32]. At the end of each experiment, mice were killed with an overdose of pentobarbital (260 mg/kg).

\section{Statistical Analysis}

Statistical analysis was performed with SYSTAT (Systat, Evanston, IL). A single-factor analysis of variance, followed by a post-hoc Tukey test, was used to determine statistical differences between means. The null hypothesis was rejected at a significance level of $P=0.05$. All means were calculated from three experiments and error bars represent standard deviations. All Western blot, activity gel, and gel mobility shift assays were repeated at least twice.

\section{RESULTS}

Increased REC Clonogenic Ability in Soft Agar After v-Ha-ras Overexpression

Cell anchorage-dependent growth was measured by the soft agar assay. Cell growth ability in soft agar is believed to be an in vitro indicator of the in vivo malignant phenotype. The results demonstrated that the parental REC cells and Neo vector control cells could not form colonies (Figure 1). However, three ras-overexpressing and high superoxide-producing clones, W13, U12, and U23 [24], had statistically significantly increased clonogenic ability in soft agar; the clonogenic fractions were $1.4 \%$, $2.8 \%$, and $2.0 \%$, respectively. These data suggest

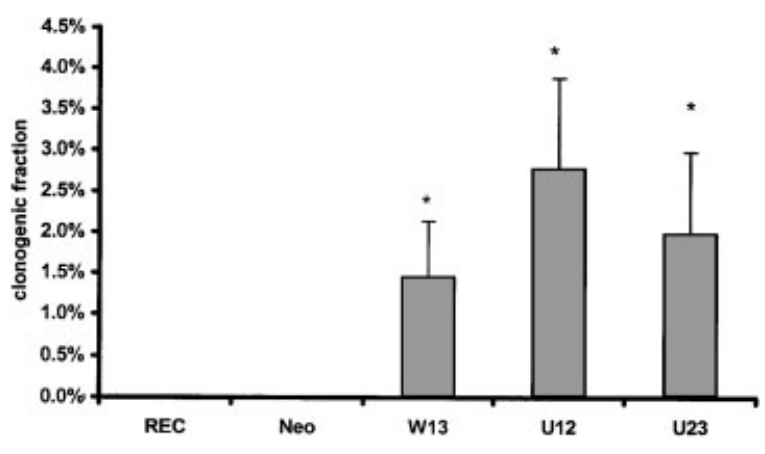

Figure 1. v-Ha-ras overexpression increased REC clonogenic ability in soft agar. The cell suspension was mixed with $3 \mathrm{~mL}$ of medium containing $0.3 \%$ agar to yield a total cell number of 2000 cells per dish. Cells were then transferred into four replicate $6-\mathrm{mm}$ tissue culture dishes containing a layer of $3 \mathrm{~mL}$ of $0.5 \%$ agar-tissue culture medium that had been prepared and solidified at $4^{\circ} \mathrm{C}$ before the experiments. Cells were incubated in a $37^{\circ} \mathrm{C}$ incubator with $5 \%$ $\mathrm{CO}_{2}$ and $95 \%$ air for $14-28 \mathrm{~d}$. Colonies with diameters of $\geq 1 \mathrm{~mm}$ were counted. REC and Neo formed no colonies. Means were calculated from three experiments; error bars represent standard deviations. ${ }^{*}$, statistically different from RECs at $P<0.05$. that REC and Neo cells are not malignant cells, as their growth is still anchorage dependent. By contrast, high superoxide-producing cells appear to be transformed to cancer cells; they not only grow faster than control cells [24], but also grow anchorage independently.

Increased Cellular MnSOD, CuZnSOD, and CAT Activities After AdMnSOD, AdCuZnSOD, and AdCAT Construct Transduction

To test the hypothesis that v-Ha-ras may mediate mitogenic signaling by altering cellular steady-state levels of superoxide or superoxide-derived ROS, we modified these levels by increasing cellular SOD or CAT enzyme levels. We transduced v-Haras-overexpressing U23 cells with adenovirus $S O D$ and $C A T$ constructs and then studied their effects on plating efficiency, DNA-binding activity of transcription factors, and tumor formation ability in nude mice. ras-overexpressing U23 cells were transduced by the adenovirus constructs AdMnSOD, AdCuZnSOD, or AdCAT or were double transduced with AdMnSOD and AdCAT or AdCuZnSOD and AdCAT. Transductions were generally performed at an MOI of 50, but some experiments used an MOI of 10, 20, or 50. AdLacZ (an MOI of 100) was used as control. Native gel SOD activity assays demonstrated MnSOD activity increased after AdMnSOD transduction and CuZnSOD activity increased after AdCuZnSOD transduction (Figure 2A). When AdCAT-infected cells were also transduced with AdCuZnSOD, slightly increased activity of CuZnSOD resulted compared with cells given AdCuZnSOD alone. This effect is probably the result of the known inhibition of $\mathrm{CuZnSOD}$ protein by $\mathrm{H}_{2} \mathrm{O}_{2}$. Figure $2 \mathrm{~B}$ demonstrates that cellular SOD activities increased as adenovirus $S O D$ construct MOI number was increased. A native gel CAT activity assay demonstrated that CAT increased after AdCAT transduction and also after AdCuZnSOD transduction but not after AdMnSOD transduction (Figure 2C). It was interesting that AdCuZnSOD increased CAT activity; this is probably due to the removal of superoxide radical, which is known to inactivate CAT protein. Figure 2D demonstrates that cellular CAT activities increased when the adenovirus CAT construct transfection MOI number was increased. These experiments demonstrate that we can modulate intracellular MnSOD, CuZnSOD or CAT activity by infecting cells with replication-defective recombinant adenoviruses expressing antioxidant enzymes.

Decreased Plating Efficiency of v-Ha-ras Transfected U23 Cells After SOD Overexpression

We demonstrated previously that v-Ha-ras overexpression increased REC clone formation ability, as measured by plating efficiency [24]. To test the effects of adenovirus $S O D$-expressing constructs on the ability of U23 to form clones, we performed 
A

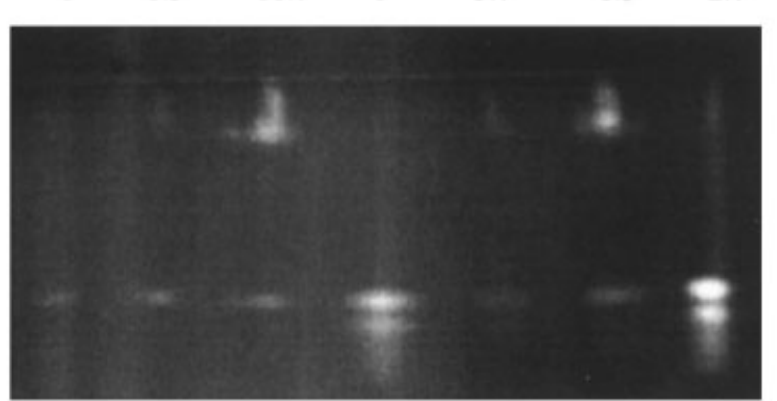

MnSOD

CuZnSOD

$\begin{array}{lllllll}1 & 1.1 & 1.6 & 5.8 & 1 & 1 & 8.1\end{array}$

B

\begin{tabular}{ccccccccc} 
U23 & AdLacZ & \multicolumn{1}{c}{ AdMn AdMn } & AdMn & AdCu AdCu & AdCu \\
& 50 & 10 & 20 & 50 & 10 & 20 & 50 \\
\hline 1 & 1 & 7 & 16.1 & 13.1 & 1 & 1 & 1
\end{tabular}

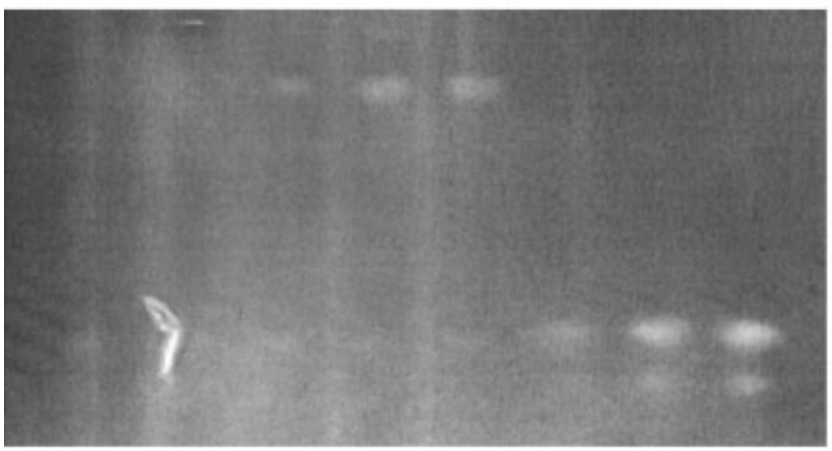

MnSOD

CuZnSOD

1

1

1

Figure 2. Adenoviral constructs increased MnSOD, CuZnSOD, and CAT activity. U23 cells were infected with adenoviruses expressing human CuZnSOD, MnSOD, or CAT CDNAs at an MOI of 50, or at the indicated $\mathrm{MOI}$ number. U23 cells transduced with an adenovirus lacZ gene construct at an $\mathrm{MOI}$ of 100 were used as a control (A and C). Cell lysates were used to run a native gel. The gels were then stained: the achromatic bands showed enzyme activity. ( $A$ and $B$ ) Native the achromatic bands showed enzyme activity. (A and B) Native $\mathrm{MOI}$ ); AdMn is AdMnSOD (MOI 50); AdCu is AdCuZnSOD (MOI 50); AdCAT is AdCAT (MOI 50); AdMC is AdMnSOD (MOI 50) + AdCAT (MOI 50): AdCC is AdCuZnSOD (MOI 50) + AdCAT (MOI 50). Increased enzymatic activities for MnSOD and CUZnSOD were found in the U23 cells transduced by AdMnSOD and AdCuZnSOD constructs, respectively. (A) Numbers at the top of the gel indicate the fold increases in MnSOD activity relative to U23 cells, as measured by densitometric analysis. Numbers at the bottom of the gel indicate

plating efficiency assays. v-Ha-ras-overexpressing U23 cells were infected by adenovirus that carried antioxidant enzyme genes or lacZ gene; trypan blue staining showed that all adenovirus-transduced U23 lines were more than $98 \%$ viable (data not shown). Figure 3 demonstrates that the plating efficiency of

\section{$\begin{array}{lll}4.1 & 11.2 & 16.7\end{array}$}

the fold increases in CuZnSOD activity relative to U23 cells, as measured by densitometric analysis. (B) Numbers directly under the lane labels indicate the calculated MOI. Numbers at the top of the ge directly under the dotted line indicate the fold increases in MnSOD activity relative to $\mathrm{U} 23$ cells, as measured by densitometric analysis. Numbers at the bottom of the gel indicate fold increases in CuZnSOD activity relative to $\mathrm{U} 23$ cells, as measured by densitometric analysis. (C and D) Native activity gel for CAT. Increased enzymatic activity was found for U23 cells transduced by AdCAT. The lanes are labeled as in panel A. (C) Numbers below the gel indicate the fold increases in CAT activity relative to $\mathrm{U} 23$ cells, as measured by densitometry. (D) Numbers at the top under the lane labels indicate the calculated MOI. Numbers at the bottom of the gel indicate the fold increases in CAT activity relative to $\mathrm{U} 23$ cells, as measured by densitometry.

U23 cells was greatly decreased after transduction of AdMnSOD at an MOI of $50(4.3 \%, P<0.05)$ or AdCuZnSOD at an MOI of $50(6.0 \%, P<0.05)$ compared with U23 cells transduced with AdLacZ at an MOI of 100 (36.3\%). However, U23 cells' plating efficiency showed no significant change 
C

U23 AdLacZ AdMn AdCu AdCAT AdMC AdCC

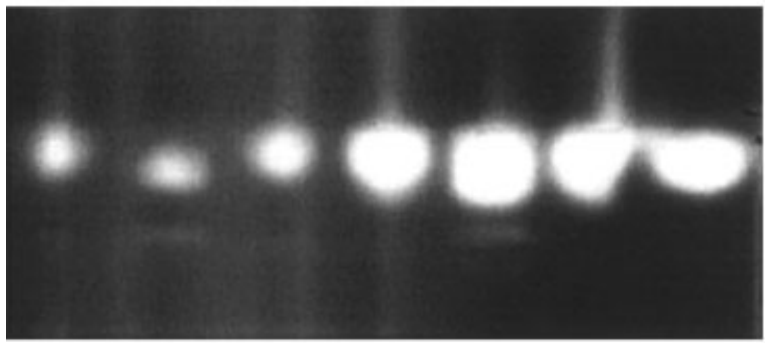

CAT

$\begin{array}{lllllll}1 & 1.1 & 1.2 & 2.5 & 3.4 & 2.4 & 2.6\end{array}$

D

U23 AdLacZ AdCAT AdCAT AdCAT

$\begin{array}{llll}50 & 10 & 20 & 50\end{array}$

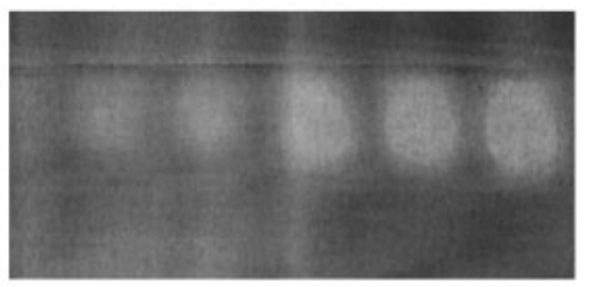

CAT

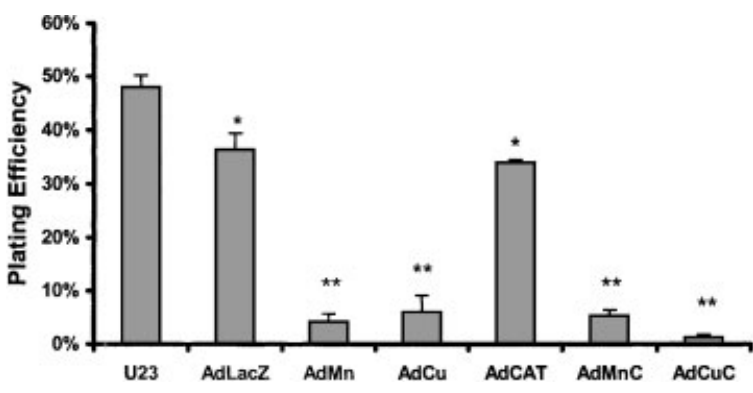

Figure 3. U23 plating efficiency was decreased by MnSOD or CuZnSOD overexpression. U23 was first transduced with adenovirus SOD or CAT constructs at an $\mathrm{MOI}$ of 50; cells were then seeded, and a cell plating efficiency assay was performed. Means were calculated from three experiments; error bars represent standard deviation. * Significantly different from U23 transduced with AdLacZ (50 MOI) at $P<0.05 ;{ }^{*}$, significantly different from ${ }^{*}$ at $P<0.05$. after transduction of AdCAT at an MOI of 50 $(34.0 \%)$ compared with that after transduction of AdLacZat an MOI of 100. Double transduction of AdMnSOD (MOI of 50) plus AdCAT (MOI of 50) or AdCuZnSOD (MOI of 50) plus AdCAT (MOI of 50) did not release the inhibition of plating efficiency caused by MnSOD or CuZnSOD. These data imply that superoxide plays a more important role than hydrogen peroxide in $\mathrm{v}$-Ha-ras-enhanced plating efficiency or that the location of CAT in peroxisomes prevents the protein from efficiently scavenging the peroxide produced by ras. Plating efficiency of U23 cells transfected by $\mathrm{AdLaCZ}$ was lower compared with that of the control U23 cells; the reason for this is unknown, but it could be due to adenovirus toxicity. However, trypan blue staining showed that all groups were greater than 98\% viable, making any effect due to toxicity unlikely. 
Table 1. Tumor Incidence at Day 5 After Cell Injection in Nude Mice

\begin{tabular}{lcccccc}
\hline & REC & Neo & U23 & U23 & U23 & U23 \\
\hline Injected cell no. $\left(\times 10^{6}\right)$ & 5.0 & 5.0 & 0.1 & 0.5 & 1.0 & 2.0 \\
Tumor incidence & $0 \%(0 / 2)$ & $0 \%(0 / 2)$ & $50 \%(1 / 2)$ & $100 \%(2 / 2)$ & $100 \%(2 / 2)$ & $100 \%(2 / 2)$ \\
\hline
\end{tabular}

Suppressed Tumor Growth in Nude Mice of v-Ha-ras-Transfected U23 Cells After SOD Overexpression

An in vivo tumorigenicity study was performed in nude mice. To determine the cell number required for tumor formation in nude mice, we used five groups of mice; each group had two animals. Each animal was injected with the following type and number of cells: REC $\left(5 \times 10^{6}\right)$, Neo $\left(5 \times 10^{6}\right)$, U23 $\left(0.1 \times 10^{6}, 0.5 \times 10^{6}, 1 \times 10^{6}\right.$ and $\left.2 \times 10^{6}\right)$. Tumors never formed in the REC or Neo groups of nude mice. However, tumors were found in U23 groups injected with $0.5 \times 10^{6}$ or more cells at $3 \mathrm{~d}$ after injection; after $5 \mathrm{~d}$, all mice from the U23 groups of nude mice had tumors (Table 1 ). Because U23 cells were strongly malignant, the tumors grew very quickly and the mice had to be killed after about 1 week. These data show that the ras-overexpressing and high superoxide-producing U23 cells have great ability to form malignant tumors in vivo. However, parental cells and vector control cells did not have this ability; they did not form tumors even when injected with 10 times the number of cells compared with U23 cells.

To test the effects of overexpressing MnSOD, CuZnSOD, or CAT on tumor formation in nude mice, we treated U23 cells with adenovirus constructs in vitro and then harvested the cells and injected them into nude mice. Trypan blue staining demonstrated that more than $98 \%$ harvested cells were viable. Mean tumor volume measurements were recorded over the 8 -d experimental period after subcutaneous injection of $0.5 \times 10^{6}$ control cells (REC and Neo) or transduced U23 cells (AdLacZ, AdMnSOD, AdCuZnSOD, AdCAT, double transfection of AdMnSOD + AdCAT or AdCuZnSOD + Ad$C A T)$. Each was at an MOI of 20. The results are shown in Figure 4. Parental RECs and Neo vector control cells had no tumor-forming ability in nude mice. U23 grew at a very fast rate in nude mice. AdLacZ- and AdCAT-transfected U23 cells had nearly the same tumor growth rate in nude mice as parental U23 cells. AdMnSOD- or AdCuZnSOD transduced $\mathrm{U} 23$ cells showed tumor growth retardation, with AdCuZnSOD being more effective than AdMnSOD. Double transduction of U23 cells with AdMnSOD plus AdCAT or AdCuZnSOD plus AdCAT led to greater inhibition of tumor growth than with either agent alone. These results suggest that superoxide may be involved in v-Ha-ras mitogenic signaling because SOD alone but not CAT alone inhibited growth. As discussed earlier, CAT may not inhibit in these experiments because it is in the wrong location.

\section{Higher Levels of MnSOD, CUZnSOD, and CAT in Tumor Tissues}

Neither MnSOD nor CuZnSOD completely inhibited U23 tumor formation in nude mice. One reason could be that the calculated MOI is only an average

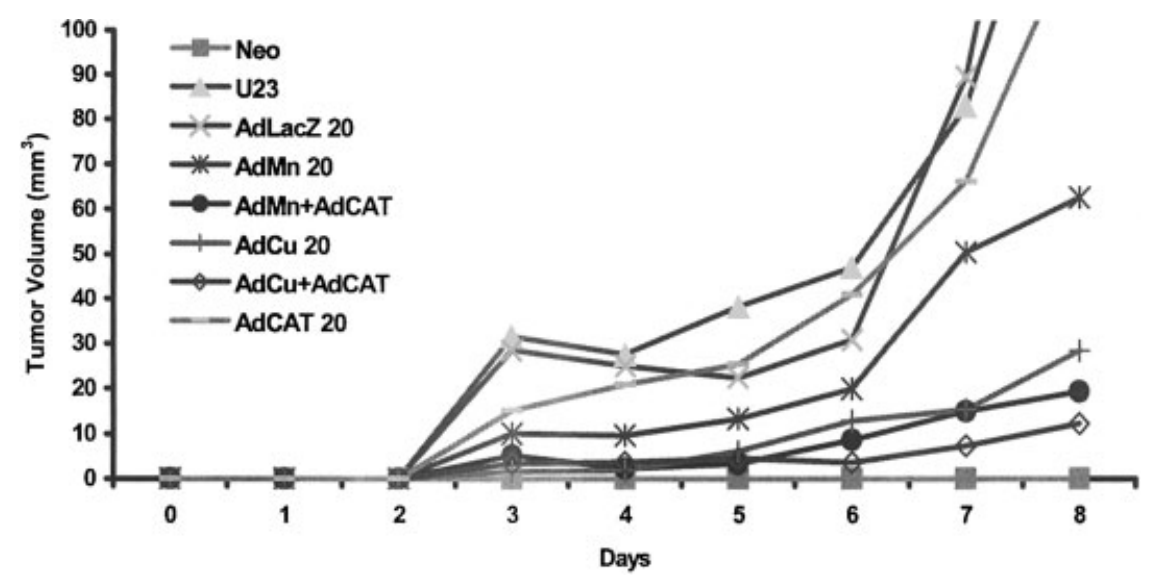

Figure 4. Effect of MnSOD, CuZnSOD, or CAT overexpression on in vivo tumor formation in nude mice. U23 cells overexpressing MnSOD or CUZnSOD by adenovirus infection at an MOI of 20 demonstrated great inhibition or delayed onset of tumor formation in nude mice. Because neither RECs nor Neo cells formed tumors, only the Neo cells are shown, as the points fall on top of each other. Mean tumor volume from four nude mice of each group is shown. 
number for each cell. Some cells could get very low copy numbers or even no infection by the adenovirus constructs. Therefore, tumors could be generated from those cells not overexpressing SOD. To test this hypothesis, we examined the tumor tissues that originated from U23 cells transduced by AdLacZ, AdMnSOD, AdCuZnSOD, or AdCAT at an MOI of 20 after the mice were euthanized at the end of the experimental period. Tumor tissues were tested for protein levels of MnSOD, CuZnSOD, and CAT. For the Western blotting assay, $100 \mu \mathrm{g}$ of cell lysate was used. Figure 5 demonstrates that tumor cells derived from U23 cells transfected by AdMnSOD or AdCAT still had several-fold higher MnSOD and CAT protein levels than the control tumors that originated from U23 cells or U23 cells transfected by AdLacZ. Tumors from U23 cells transduced with AdCuZnSOD had slightly more CuZnSOD protein than the control tumors. These data show that at least some of the tumor tissue was overexpressing SOD and that the tumor growth may not be entirely the result of cells losing the overexpression of SOD.

Altered DNA-Binding Activities of Activator Protein 1 $(\mathrm{AP}-1)$ and Nuclear Factor $\kappa \mathrm{B}(\mathrm{NF}-\kappa \mathrm{B})$ in SOD-Overexpressing Cells

Figure 6 shows the results of gel-shift experiments measuring DNA-binding activity of AP-1 and NF- $\mathrm{B}$ after U23 cells were treated with the antioxidant NAC (10 mM) or the adenovirus constructs AdLacZ,

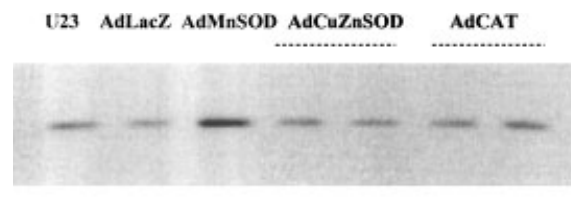

MnSOD

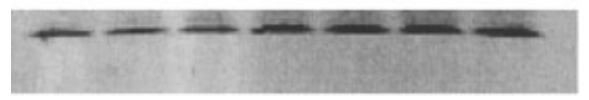

CuZnSOD

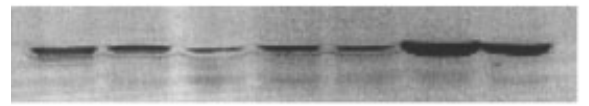

CAT

Figure 5. Tumor tissues from nude mice still had increased levels of MnSOD and CAT but little change in CUZnSOD. Nude mice were injected with U23 cells that were transduced by AdLacZ, AdMnSOD, AdCUZnSOD, or AdCAT at an MOI of 50. After the nude mice were killed on the 10th day, their tumor tissues were isolated. The tumor tissues were tested for protein levels of MnSOD, CuZnSOD, and CAT by Western blotting. The tumor tissues were homogenized; protein concentrations were determined with the Bio-Rad Bradford dye binding protein assay kit; and $100-\mu \mathrm{g}$ cell lysates were used for Western blotting. Tumors were from lane 1, U23 cells; lane 2, U23 cells treated with AdLacZ; lane 3, U23 cells treated with AdMnSOD; lanes 4 and 5, U23 cells treated with AdCUZnSOD; lanes 6 and 7, U23 cells treated with AdCAT.
AdMnSOD, AdCuZnSOD, or AdCAT at an MOI of 50. A gel mobility shift assay demonstrated that preincubating U23 nuclear protein with antibody against c-jun/AP-1 lowered AP-1 binding. AdLacZ, AdCuZnSOD, or AdCAT infection did not change AP-1 DNA-binding activity in U23 cells. In contrast, transduction with AdMnSOD caused an increase in the AP-1 DNA-binding activity (Figure 5A). Previous work demonstrated the specificity of this assay using unlabeled AP-1, AP-2, and NF- $\kappa$ B oligos [24]. Similar results were found in NF- $\kappa B$ DNA-binding activity experiments (Figure 5B). Pre-incubating U23 nuclear protein with anti-p50 (NF- $\mathrm{BB})$ antibody lowered NF- $\kappa$ B binding. Both AP-1 and NF- $\kappa \mathrm{B}$ DNA-binding activity were blocked by NAC treatment, suggesting that high oxidant levels can activate these transcription factors. Interestingly, the mobilities of both the AP- 1 and NF- $\kappa \mathrm{B}$ complexes appeared to be altered in the AdMnSODinfected cells. Although the significance of this apparent change in mobility is not entirely clear, one possibility is that new heterodimeric forms of AP- 1 and NF- $\kappa \mathrm{B}$ might be induced to form under the conditions imposed by MnSOD overexpression. This can be tested in the future by gel supershift experiments in which nuclear extracts are treated with antibodies against the other AP-1 family members. Both AP-1 and NF- $\kappa$ B include several subunit members, most of which are able to homodimerize and heterodimerize, resulting in complexes of distinct DNA binding specificity with different cellular functions $[33,34]$. Therefore, we hypothesize that different AP- 1 or NF- $\mathrm{BB}$ dimers will induce different genes to be expressed; it is possible that the new activated AP-1 or NF- $\mathrm{KB}$ dimers can induce genes to produce the MnSOD tumor suppression effects. In the AP- 1 family, jun $\mathrm{D}$ can slow cellular proliferation in fibroblasts [33]; in the NF- $\mathrm{KB}$ family, p50 and p52 homodimers can act primarily as repressors [34].

Increased Intracellular GPx1 Activity After Infection of U23 Cells with Adenovirus GPX1 Constructs

GPx1 is another important primary antioxidant enzyme that contributes to the cellular oxidant levels through its modulation of $\mathrm{H}_{2} \mathrm{O}_{2}$ levels. GPx1 is found in the cytoplasm, nucleus, and mitochondria [30]. We wanted to determine whether removal of peroxide in these locations affected the actions of ras. The above results showed that CAT, which is found in peroxisomes, did not affect ras-induced transformation. In order to modify the cellular GPx1 activity, U23 cells were transiently transduced with adenovirus constructs of human GPX1 cDNAs at MOIs of $10,20,50$, or 100, and trypan blue staining showed that more than $98 \%$ cells were viable (data not shown). U23 cells infected by an adenovirus lacZ gene construct at an MOI of 20 were used as a control. Native activity gel immunoblotting 
A

\section{Probe U23 Ab NAC AdLacZ AdMn AdCu AdCAT}

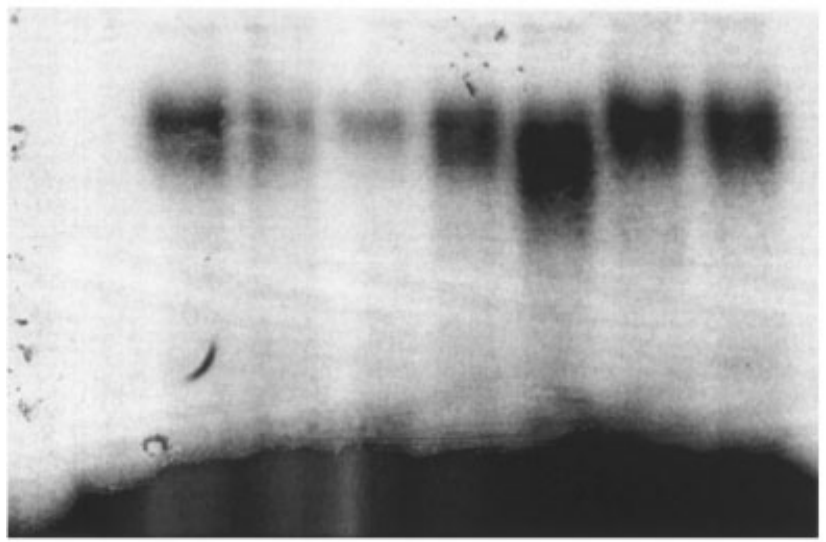

\section{AP-1}

B

Probe U23 Ab NAC AdLacZ AdMn AdCu AdCAT

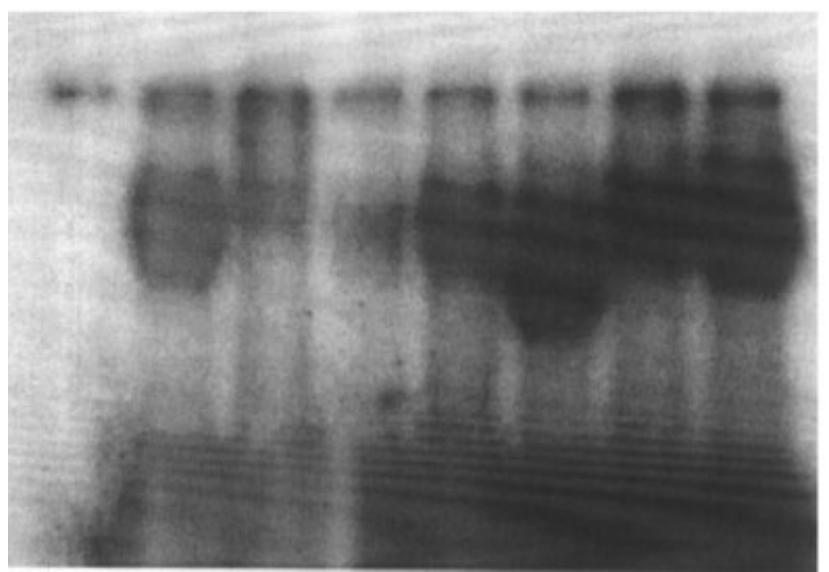

Figure 6. SOD overexpression modified U23 cell AP-1 and NF- $\kappa$ B DNA-binding activity. (A) AP-1 DNA-binding activity was changed after SOD overexpression in U23 cells. (B) NF-KB DNA-binding activity was changed after SOD overexpression in U23 cells. Ab, antibody to

NF-kB anti-p50 (NF-kB). U23 cells were treated NAC or with adenovirus laCZ, MnSOD, CUZnSOD or CAT constructs at an $\mathrm{MOI}$ of 20, after which nuclear protein was extracted. One and a half micrograms of nuclear protein for each sample was incubated with ${ }^{32} \mathrm{P}$-labeled oligo probes (for NF- $\kappa B$ or $A P-1$ ), in the presence of $1 \mu \mathrm{g}$ of poly (dldC) and $1 \times$ gel shift buffer at room temperature for $15 \mathrm{~min}$. Binding reactions were loaded on $5 \%$ polyacrylamide gels and run at $35 \mathrm{~mA}$ for $40 \mathrm{~min}$ in $1 \times$ TBE buffer. The gel was wrapped in a sheet of plastic and was exposed to $X$-ray film overnight at $-80^{\circ} \mathrm{C}$.

assay was performed to test the GPx1 protein levels in these cells. Figure 7A shows that GPx1 immunoreactive protein level increased after AdGPX1 infection and increased with MOI. GPx1 enzymatic activities were also measured by a native gel activity assay. Figure 7B demonstrates that GPx1 activity increased in the AdGPX1-transduced U23 cells, and increased activity was found with increasing MOI. These results show that we can increase cellular GPx1 activity level by transduction of adenovirus GPX1 construct.

Suppressed Tumor Growth in Nude Mice of v-Ha-ras Transfected U23 Cells After GPX1 Overexpression

An in vivo tumorigenicity study was performed in nude mice. U23 cells were treated with adenovirus constructs in vitro and then injected into nude mice. Mean tumor volume measurements over the 10-d experimental period after subcutaneous injection of $0.2 \times 10^{6}$ control cells (REC and Neo) or transduced U23 cells (AdLacZ, AdMnSOD, AdCuZnSOD, AdCAT, AdGPX1, two combinations of AdMnSOD + AdGPx1 or AdCuZnSOD + AdGPx1, all at an MOI of 50) are shown in Figure 8 . The data show that U23 cells transduced by AdLacZ had almost the same tumor growth rate in nude mice as U23 cells, and U23 cells transduced with AdCAT showed a small inhibition of growth compared with control cells (U23 and U23 cells transduced by $\mathrm{Ad} L a c Z$ ). U23 cells transduced by AdMnSOD or AdCuZnSOD showed greater tumor growth retardation; the tumors grew more slowly than U23 cells and $\mathrm{U} 23$ cells transduced by $\mathrm{Ad} L a c Z$ and also more slowly than U23 cells transduced by AdCAT. To our 
A

Pure Empty U23 AdLacZ AdGPx AdGPx AdGPx AdGPx

GPx

20

20

50

100

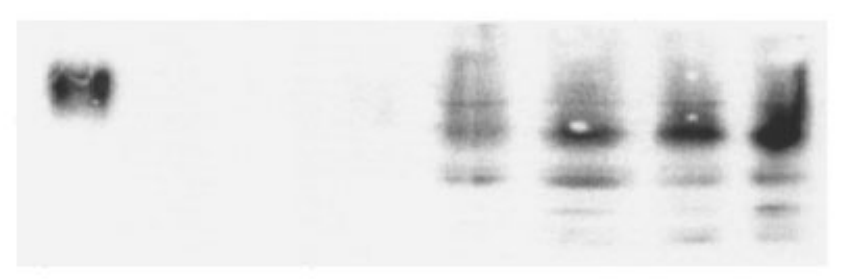

\section{GPx1}

B
Pure
GPx 20 10
U23 AdLacZ AdGPx AdGPx AdGPx AdGPx

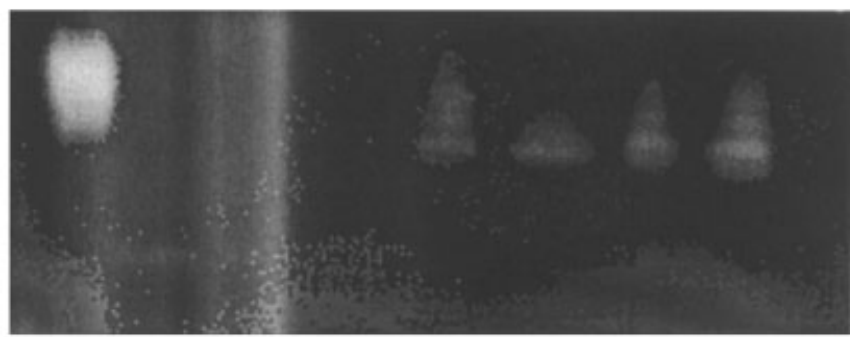

\section{GPx1}

Figure 7. Intracellular GPx1 activity was modified by transduction of adenovirus GPX1 constructs into U23 cells. U23 cells were infected with adenovirus constructs of human GPX1 CDNAs at MOls of $10,20,50$, or 100 . U23 cells transduced by an adenovirus $L a C Z$ gene construct at an $\mathrm{MOI}$ of 20 were used as a control. (A) GPx1 protein leve increased after AdGPX1 transduction. Cell lysates, $400 \mu \mathrm{g}$, were used to run an immunoblotting assay for GPx1. Numbers at the top of the blot under the lane labels are the calculated MOls. Numbers at the bottom of the blot are the fold increases in immunoreactive protein relative to $\mathrm{U} 23$ cells, as measured by densitometry. Lane 1, bovine GPx 1 protein control; lane 2, empty (no sample was added here); lane 3, sample from U23 cells; lane 4 sample from U23 cells transduced with AdLaCZ at $20 \mathrm{MOl}$; lanes 5-8, samples from U23 cells transduced by AdGPX1 at different MOls; lane 5, $\mathrm{MOI}$ of 10; lane $6, \mathrm{MOI}$ of 20; lane 7, $\mathrm{MOI}$ of 50 lane $8, \mathrm{MOI}$ of 100 . (B) Intracellular GPx1 activity increased after AdGPX1 transduction. 400- $\mu \mathrm{g}$ cell lysates were used to run a native gel. The gels were then stained; the achromatic bands show the enzyme activities. Again, the numbers at the top of the gel under the lane labels indicate the calculated MOls and the numbers at the bottom of the gel indicate the fold increases in GPx1 activity relative to $\mathrm{U} 23$ cells, as measured by densitometry.

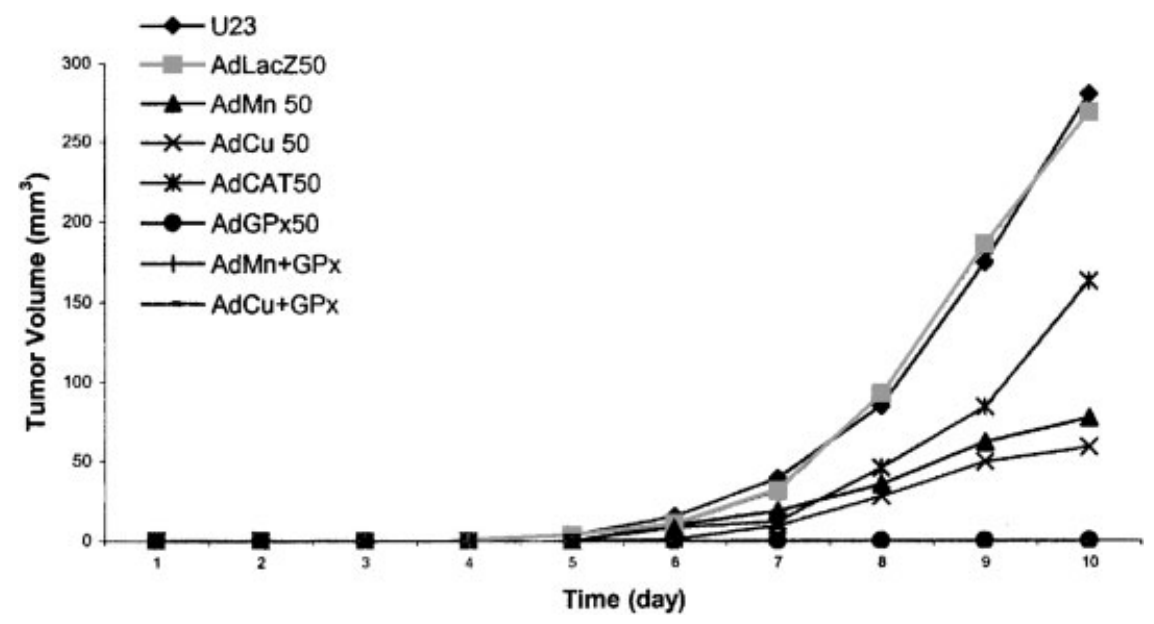

Figure 8. Effect of GPx1 overexpression on tumor formation in nude mice. U23 cells overexpressing GPx1 by adenovirus GPX1 constructs at an $\mathrm{MOI}$ of 50 demonstrated no tumor formation in nude mice. Mean tumor volumes from four nude mice of each group are shown. The AdGPx50, AdMn $+G P x$, and AdCu+GPx groups did not form tumors; thus, all points fall on top of each other at the bottom of the graph on the $x$-axis. Cells were prepared with serum-free Dulbecco's modified Eagle's high-glucose medium with the indicated cell number $(0.1 \mathrm{ml})$ and were injected into the left rear leg of each nude mouse. 
surprise, no tumor formation was observed in U23 cells transduced by AdGPX1, neither in mice injected with U23 cells transduced by AdGPx1 single transduction nor by double transduction with AdGPX1 and AdMnSOD or AdCuZnSOD. This result suggests that increased cellular GPx1 activity can completely block ras mitogenic signaling from superoxide overproduction, and superoxide overproduction can maintain a relatively high concentration of $\mathrm{H}_{2} \mathrm{O}_{2}$ or other hydroperoxides, which is needed for superoxide to act as a second messenger. Removing $\mathrm{H}_{2} \mathrm{O}_{2}$ by increased cellular GPx1 activity may abolish the ras-rac superoxide pathway. This could be the reason that U23 cells transduced by AdGP 1 had no tumor-forming ability in nude mice.

\section{DISCUSSION}

The proto-oncogene c-Ha-ras is frequently mutated in human tumors and functions to stimulate signal transduction cascades chronically, resulting in various cellular processes $[35,36]$. We studied v-Ha-ras-transfected rat kidney epithelial cells and three prototypical clonal cell lines, W13, U12, and U23, which stably express different v-Ha-ras protein levels; these lines have been extensively characterized [24]. These v-Ha-ras-transfected cells overproduced superoxide, and it was found that superoxide production could be inhibited by treatment with inhibitors of ras maturation; these cells had a shortened cell doubling time and increased plating efficiency in normal medium. In the present report, we demonstrated that the v-Ha-ras-transfected RECs became malignant, grew in soft agar, and also formed tumors in nude mice. Parental cells and vector control cells did not have these abilities. Furthermore, overexpression of SOD and GPx1 in the U23 cell line could suppress its malignant tumor phenotype. Therefore, we hypothesize that superoxide and its derived ROS act as second messengers in ras mitogenic signal transduction.

At least two pathways are associated with ras mitogenic signal transduction. One is the ras-raf pathway $[37,38]$. ras can switch on and promote the activity of raf, a kinase, which in turn triggers the activation of mitogen-activated protein kinase. The phosphorylated mitogen-activated protein kinase further modulates the activity of transcription factors and, ultimately, cell proliferation. The other pathway is the newly emerged ras-rac superoxide pathway. ras can activate rac, another GTPbinding protein, which triggers the assembly of the NADPH oxidase system to generate superoxide. Superoxide or superoxide-derived ROS modulates the cellular redox environment, which plays a direct role in the signaling cascade that underlies mitogenesis and/or transformation [39]. Evidence has accumulated over the past few years that the ras-rac superoxide pathway may play a more important role in ras mitogenic signal transduction. NIH/3T3 cells transformed with Ha-ras V12 produce superoxide constitutively, and such production is required for their unchecked proliferation [22]. It has also been shown that overexpression of nox 1 (formerly called mox1), which encodes a homologue of the catalytic subunit of the superoxide-generating NADPH oxidase of phagocytes, in NIH/3T3 cells increases superoxide generation and cell growth [40]. Cells overexpressing nox1 have a transformed appearance, show anchorage-independent growth, and produce tumors in athymic mice. Thus, overexpression of either ras or nox 1 in immortal cells will lead to production of superoxide, increased cell growth, and expression of the malignant phenotype.

Our data also showed that overexpression of either MnSOD or CuZnSOD to remove superoxide suppressed the cellular malignant phenotype, as shown by decreasing plating efficiency in normal medium and tumor formation in nude mice. CuZnSOD is located in the cytoplasm; MnSOD is located in the mitochondrial matrix. In the v-Haras-overexpressing cells, superoxide may possibly come from the non-phagocytic-like NADPH oxidase nox $1[24,40]$ in the plasma membrane. We first thought that the $S O D$ tumor-suppressive effect was probably not from the accumulated high steadystate concentration of hydrogen peroxide, because the cotransduction of adenovirus $\mathrm{MnSOD}$ or $\mathrm{CuZn-}$ $S O D$ constructs with adenovirus CAT constructs, whose product, CAT, removes toxic $\mathrm{H}_{2} \mathrm{O}_{2}$, failed to rescue the cells. Indeed, AdCAT appeared to enhance the growth-suppressive effect of AdSOD. Moreover, transduction of CAT alone at an MOI of 50 appeared to have a growth-suppressive effect (Figure 8), whereas transduction at a lower MOI showed little growth-suppressive effect (Figure 4). However, CAT is located primarily in peroxisomes, so it may not be in a good location to remove $\mathrm{H}_{2} \mathrm{O}_{2}$ generated from either SOD. GPx1 may remove $\mathrm{H}_{2} \mathrm{O}_{2}$ produced from ras activation more effectively than CAT because it is located in the cytoplasm and can work at lower $\mathrm{H}_{2} \mathrm{O}_{2}$ concentration. Moreover, GPx1 not only works on $\mathrm{H}_{2} \mathrm{O}_{2}$ but also on lipid peroxides.

Neither MnSOD nor CuZnSOD completely inhibited U23 tumor formation in nude mice. One reason could be that the calculated MOI is only an average number for each cell. Some cells could get a very low copy number or even not be transduced by the adenovirus constructs. Therefore, tumors could be generated from such cells. Another reason could be the ras-raf pathway might still be activated in the SOD-overexpressing U23 cells, even though the rasrac superoxide pathway is blocked by SOD overexpression. Tumor tissue from U23 cells transfected with AdCuZnSOD constructs had only slightly elevated CuZnSOD protein levels (Figure 5). Thus, the tumor could originate from U23 with low MOI or even with no transduction by AdCuZnSOD constructs. The tumor could also originate from cells that 
originally had high CuZnSOD levels but that lost expression of the protein with time. However, tumor tissue from U23 cells transduced by AdMnSOD constructs still had relatively high MnSOD protein levels. Thus, the tumor may have originated in a cell with high MnSOD levels, or it may have come from cells that lost MnSOD expression. It appears that, in this case, MnSOD is less effective in suppressing tumor formation than CuZnSOD; the reason could be that MnSOD, located in the mitochondria, is less effective in scavenging superoxide generated from ras activation than CuZnSOD, located in the cytoplasm. However, it is very difficult to determine which protein is a more effective tumor suppressor. Even though both proteins were compared at equivalent MOIs, the same MOI of each construct will most likely produce different induction of the respective enzymes. Moreover, because endogenous levels of the enzymes may be different, the fold increase after transduction may be different. Thus, it is difficult to determine which protein is more effective. This difficulty is illustrated by the different results in Figures 4 and 8 . When MnSOD and CuZnSOD were transduced at an MOI of 20 (Figure 4), CuZnSOD was more effective than MnSOD. However, when both were transduced at an MOI of 50 (Figure 8), similar growth inhibitory effects resulted with both proteins. Likewise, GPx1 appeared to be the most effective of the antioxidant proteins tested at inhibiting the malignant phenotype, but, again, this could be because relatively more protein was produced.

It is not surprising to find that v-Ha-ras-transfected RECs had enhanced DNA-binding activity of transcription factors, including NF- $\kappa \mathrm{B}, \mathrm{AP}-1$, and AP-2, which can change gene expression and lead to cell proliferation [24]. However, it is interesting to find that MnSOD overexpression in U23 clones might be able to induce DNA-binding activity of a new heterodimer of NF- $\kappa$ B or AP-1. NF- $\kappa$ B or AP-1 are in a protein family that includes various protein members. NF- $\kappa \mathrm{B}$ or AP-1 is formed by dimerizing family members; the composition of the dimer determines its DNA-binding specificity for different genes $[33,34]$. These heterodimers may be important to understanding the SOD tumor suppression effects.

Mutations in ras alleles occur in 30\% of all human tumors, making ras the most widely mutated human proto-oncogene. Superoxide and superoxide-derived ROS may mediate ras-induced cellular responses in these tumors. However, cancer cells normally have low levels of MnSOD, CuZnSOD, and CAT compared with their normal cell of origin [41]; therefore, cancer cells may maintain relatively high levels of superoxide or superoxide-derived ROS to accelerate their proliferation. This phenomenon suggests that modulation of the redox environment of the cell may provide one mechanism to explain the observation that some antioxidants appear to exert protective effects against the induction of human cancer.

\section{ACKNOWLEDGMENTS}

This research was supported by National Institutes of Health grant CA66081, to L.O., G.B., and F.D.

\section{REFERENCES}

1. Anderson MW, Reynolds SH, You M, Maronpot RM. Role of proto-oncogene activation in carcinogenesis. Environ Health Perspect 1992;98:13-24.

2. Freeman BA. Biological sites and mechanisms of free radical production. In: Armstrong D, Sohal RS, Cutler RG, Slater TF, editors. Molecular biology, aging, and disease. New York: Raven Press; 1984. p 43-52.

3. Bize IB, Oberley LW, Morris HP. Superoxide dismutase and superoxide radical in the Morris hepatomas. Cancer Res 1980;40:3686-3693.

4. Fernandez-Pol JA, Hamilton PD, Klos DJ. Correlation between the loss of the transformed phenotype and an increase in superoxide dismutase activity in a revertant subclone of sarcoma virus-infected mammalian cells. Cancer Res 1982;42:609-617.

5. Kelner M, Bagnell R. Alteration of endogenous glutathione peroxidase, manganese superoxide dismutase, and glutathione transferase activity in cells transfected with a copper-zinc superoxide dismutase expression vector. J Biol Chem 1990;265:10872-10875.

6. Oberley LW, Bize IB, Sahu SK, Leuthauser SWH, Gruber HE Superoxide dismutase activity of normal murine liver, regenerating liver, and $\mathrm{H} 6$ hepatoma. J Natl Cancer Inst 1978;61:375-379.

7. Marlens F, Nicole A, Sinet PM. Lowered level of translatable messenger RNAs for manganese superoxide dismutase in human fibroblasts transformed by SV40. Biochem Biophys Res Commun 1985;129:300-305.

8. McCormick ML, Oberley TD, Elwell JH, Oberley LW, Sun Y, $\mathrm{Li}$ JJ. Superoxide dismutase and catalase levels during estrogen-induced renal tumorigenesis, in renal tumors and their autonomous variants in the Syrian hamster. Carcinogenesis 1991;12:977-983

9. Sun $Y$, Li $Y$, Oberley LW. Superoxide dismutase activity during dimethylhydrazine colon carcinogenesis and the effects of cholic acid and indole. Free Radic Res Commun 1993:4:299-310.

10. Oberley LW, Kasemset St. Clair D, Autor A, Oberley TD. Increase in manganese superoxide dismutase activity in the mouse heart after X-irradiation. Arch Biochem Biophys 1987;254:69-80.

11. Church S, Grant JW, Ridnour LA, Oberley LW, Swanson PE. Increased manganese superoxide dismutase expression suppresses the malignant phenotype of human melanoma cells. Proc Natl Acad Sci USA 1993;90:3113-3117.

12. Li JJ, Oberley LW, St. Clair DK, Ridnour LA, Oberley TD. Phenotypic changes induced in human breast cancer cells by overexpression of manganese-containing superoxide dismutase. Oncogene 1995;10:1989-2000.

13. Liu R, Oberley TD, Oberley LW. Transfection and expression of MnSOD CDNA decreases tumor malignancy of human oral squamous carcinoma. Hum Gene Ther 1997;8:585589.

14. Yan T, Oberley LW, Zhong W, St. Clair DK. Manganesecontaining superoxide dismutase overexpression causes phenotypic reversion in SV40-transformed human lung fibroblasts. Cancer Res 1996;56:2864-2871.

15. Zhong W, Oberley LW, Oberley TD, St. Clair DK. Suppression of the malignant phenotype of human glioma cells by overexpression of manganese superoxide dismutase. Oncogene 1997;14:481-490. 
16. Ames BN. Measuring oxidative damage in humans: Relation to cancer and aging. IARC Sci Publ 1988;89:407-416.

17. Sundaresan $M, Y u Z X$, Ferrans VJ, Iran $K$, Finkel $T$. Requirement for generation of $\mathrm{H}_{2} \mathrm{O}_{2}$ for platelet-derived growth factor signal transduction. Science 1995;270:296299

18. Ohba M, Shibanuma T, Kuroki T, Nose K. Production of hydrogen peroxide by transforming growth factor-beta 1 and its involvement in induction of egr-1 in mouse osteoblastic cells. J Cell Biol 1994;126:1079-1088.

19. Meier B, Radeke HH, Selle $\mathrm{S}$, et al. Human fibroblasts release reactive oxygen species in response to interleukin-1 or tumor necrosis factor- $\alpha$. Biochem J 1989;263:539-545.

20. Lo YYC, Cruz TF. Involvement of reactive oxygen species in cytokine and growth factor induction of c-fos expression in chondrocytes. J Biol Chem 1995;270:11727-11730.

21. Levine $A$, Tenhaken $\mathrm{R}$, Dixon $\mathrm{R}$, Lamb $\mathrm{C}$. $\mathrm{H}_{2} \mathrm{O}_{2}$ from the oxidative burst orchestrates the plant hypersensitive resistance response. Cell 1994;79:583-593.

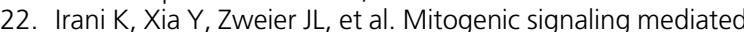
by oxidants in Ras-transformed fibroblasts. Science 1997; 275:1649-1652.

23. Yang JQ, Li SJ, Domann FE, Buettner GR, Oberley LW Superoxide generated in V-Ha-ras-transduced human keratinocyte HaCaT cells. Mol Carcinogen 1999;26:180188.

24. Yang JQ, Li S, Huang $Y$, et al. V-Ha-Ras overexpression induces superoxide production and alters levels of primary antioxidant enzymes. Antioxid Redox Signal 2001;3:697709.

25. Larco JED, Todaro GJ. Epithelioid and fibroblastic rat kidney cell clones: epidermal growth factor (EGF) receptors and the effect of mouse sarcoma virus transformation. J Cell Physiol 1978;94:335-342.

26. Beauchamp C, Fridovich I. Superoxide dismutase: Improved assay and an assay applicable to acrylamide gels. Anal Biochem 1971;44:276-287.

27. Sun Y, Elwell JH, Oberley LW. A simultaneous visualization of the antioxidant enzymes glutathione peroxidase and catalase on polyacrylamide gels. Free Radic Res Commun 1988; 5:67-75
28. Wang Q, Finer $\mathrm{MH}$. Second-generation adenovirus vectors Nature Med 1996:2:714-716.

29. Li Q, Sanlioglu S, Li S, Ritchie T, Oberley L, Engelhardt JF GPX-1 gene delivery modulates NFKB activation after diverse environmental injuries through a specific subunit of the IKK complex. Antioxid Redox Signal 2001;3:415-432.

30. Li S, Yan T, Yang J-Q, Oberley TD, Oberley LW. The role of cellular glutathione peroxidase redox regulation in the suppression of tumor cell growth by manganese superoxide dismutase. Cancer Res 2000;60:3927-3939.

31. Huang $Y$, Domann FE. Redox modulation of AP-2 DNA binding activity in vitro. Biochem Biophys Res Commun 1998;249:307-312.

32. Zhang HJ, Yan T, Oberley TD, Oberley LW. Comparison of effects of two polymorphic variants of manganese superoxide dismutase on human breast MCF-7 cancer cell phenotype. Cancer Res 1999;59:6276-6283

33. Pfarr CM, Mechta F, Spyro G, Lallemant D, Carillo S, Yaniv M. Mouse JunD negatively regulates fibroblast growth and antagonizes transformation by ras. Cell 1994;76:747-760.

34. Muller CW, Harrison SC. The structure of the NF- $\kappa B$ p50:DNA-complex: A starting point for analyzing the Re family. FEBS Lett 1995;369:113-117.

35. Barbacid M. Ras genes. Annu Rev Biochem 1987:56:779_ 827.

36. Bos JL. Ras oncogenes in human cancer: A review. Cancer Res 1989:49:4682-4689.

37. Lowy DR. Function and regulation of Ras. Annu Rev Biochem 1993;62:851-891.

38. Irani K, Goldschmidt-Clermont PJ. Ras, superoxide and signal transduction. Biochem Pharmacol 1998;55:13391346.

39. Schafer FQ, Buettner GR. Redox environment of the cell as viewed through the redox state of the glutathione disulfide/ glutathione couple. Free Radic Biol Med 2001;30:11911212.

40. Suh YA, Arnold RS, Lassegue B, et al. Cell transformation by the superoxide-generating oxidase Mox1. Nature 1999; 401:79-82.

41. Oberley LW, Buettner GR. Role of superoxide dismutase in cancer: a review. Cancer Res 1979;39:1131-1149. 